\title{
Review and Performance Comparison of Pansharpening Algorithms for RASAT Images
}

\author{
Sevcan Kahraman iD, Alp Ertürk \\ Kocaeli University Laboratory of Image and Signal Processing (KULIS), Kocaeli, Turkey
}

Cite this article as: S. Kahraman, A. Ertürk. "Review and Performance Comparison of Pansharpening Algorithms for RASAT Images". Electrica, vol. 18, no. 1, pp. 109-120, 2018.

\begin{abstract}
This study presents the most extensive performance comparison of pansharpening methodologies by considering 17 pansharpening algorithms that are applied to the satellite images obtained from RASAT, which is the first earth observation satellite designed and manufactured in Turkey. Standard and state-of-the-art pansharpening approaches from various categories, such as component substitution (CS), modulation based (MB), multiresolution analysis (MRA), and hybrid and variational methods, are included in order to gain a better insight and perform a thorough analysis of the performance of various pansharpening methods. The experimental validation procedure was designed according to Wald's protocol, and the performance evaluations were conducted both qualitatively and quantitatively on the basis of seven quantitative evaluation criteria.
\end{abstract}

Keywords: RASAT, pansharpening, performance, review

Address for Correspondence:

Sevcan Kahraman

E-mail:

sevcan.kahraman@kocaeli.edu.tr

Received: 05.06.2017

Accepted: 21.09 .2017

C Copyright 2018 by Electrica

Available online at

http://dergipark.gov.tr/iujeee

DOI: 10.5152/iujeee.2018.1817

\section{Introduction}

Image fusion is the process of combining relevant information from multitude images, often acquired by different sensors and imaging technologies. The most common approach is the fusion of a high spatial resolution image with a high spectral resolution image. If the high spatial resolution image is a panchromatic image, then the fusion process is called pansharpening.

Pansharpening, in its original form, is the process which combines multispectral (MS) images with high spatial resolution panchromatic (PAN) images in order to construct high spatial resolution MS images. Pansharpening has long been an important field of research because it enables the construction of images with high spatial and high spectral resolution, which is not directly obtainable from imaging sensors due to various technical limitations. Pansharpening was originally applied to PAN an MS images, but have evolved to include fusion of PAN and hyperspectral (HS) images, and MS and HS images, among others. At its basis, pansharpening is the process of extracting spatial information from one image and injecting this information to the other image in order to enhance spatial resolution.

There are various pansharpening reviews in the literature. In [1], the pansharpening performance of four pan-sharpening approaches have been compared and it is stated that context-based decision approach gives the best result. In [2], it has been demonstrated that methods in accordance with the Amélioration de la Résolution Spatiale par Injection de Structures (Improving Spatial Resolution by Structure Injection-ARSIS) concept prevent spectral distortion for pansharpened images. In [3], performances of the pansharpening algorithms in commercial software packages have been compared for images acquired from IKONOS, QuickBird, GeoEye-1, and WorldView-2 satellites, and the best fusion quality is reported by principal component analysis (PCA). In [4], eight pansharpening approaches are compared for performance with several spatial and spectral quality indexes, and it has been stated that approaches combining A Tróus wavelet transform with Intensity-Hue-Saturation (IHS) trans- 
form and PCA provide the best spatial-spectral tradeoff. In [5], advanced pansharpening approaches such as Generalized Laplacian Pyramid with Modulation Transfer Function (MTFmatched filter) and Context-Based Decision injection scheme (MTF-GLP-CBD) have been applied to datasets acquired by IKONOS-2 and WorldView-2 satellites, and best pansharpening results are obtained from Gaussian MTF-matched filter with High Pass Modulation injection model (MTF-GLP-HPM) method. In a recent review, eleven different state-of-the-art pansharpening methods are adapted to the HS images, and Bayesian Sparse approach has provided the best performance for hyperspectral pansharpening [6]. In [7], pansharpening approaches in the literature are classified into different categories according to the main technique they use by Amro and et al. In study [8], twenty one pansharpening techniques are presented and evaluated for the VNIR and SWIR bands of Sentinel-2 and MTF-GLP-CBD provides the best consistency pansharpened result.

This paper is concerned with pansharpening performances for RASAT images. RASAT is the first earth observation satellite designed and produced in Turkey by TÜBITAK-UZAY (TÜBITAK Space Technologies Research Institute). RASAT has been in operation since 2011, and is the second remote sensing imaging satellite of Turkey after BILSAT. RASAT provides 7.5 meter spatial resolution PAN and 15 meter spatial resolution RGB images. There are some works on the literature on pansharpening for RASAT images. In [9], nine different pansharpening approaches are evaluated for RASAT images by utilizing six assessment quality metrics, and Hyperspherical Color Space (HCS) and High Pass Filter (HPF) methods are shown to provide the best performances. In [10], GPU speed-up is assessed for pansharpening on RASAT images. In [11], automatic image processing workflow is drawn for RASAT and GÖKTÜRK-2 satellite images. In [12], the performance evaluation of IHS, Brovey and PCA pansharpening methods are evaluated for RASAT and GÖKTÜRK-2 images.

In this paper, a wide range of pansharpening methods, both benchmark and state-of-the-art, are evaluated and compared for performance on RASAT images. The evaluation is performed over several images, both visually and quantitatively, by utilizing a wide array of metrics, i.e. root mean squared error (RMSE), correlation coefficient (CC), spectral angular mapper (SAM), Erreur Relative Globale Adimensionnelle de Synthese (ERGAS), peak signal-to-noise ratio (PSNR), structural similarity index (SSIM), and universal image quality index (UIQI). This work's main contribution is the utilization of the largest number of pansharpening approaches and performance metrics used up-to-date in any pansharpening review work in the literature.

The paper is organized as follows. Section 2 summarizes and explains the pansharpening approaches. The experimental results obtained on various RASAT images are provided in Section 3. The paper is concluded in Section 4 with some possible future study lines.

\section{Review of Pansharpening Approaches}

The aim of the pansharpening process is the integration of high frequency information, which is extracted from the PAN image, with the lower level spatial information from the MS image in order to obtain high spatial resolution MS image.

Although there is no universal classification, pansharpening approaches can be basically grouped into five categories according to fusion mechanism. These are component substitution (CS), modulation based (MB), multi-resolution analysis (MRA), hybrid methods and variational approaches. These categories and the respective methods in each category that are used in this paper are explained below.

\section{Component Substitution (CS) Methods}

\section{CS approach consists of three steps:}

1. MS image is upsampled to the resolution of PAN image, and transformed to a new space. This new space aims to separate the spectral and spatial information, which will be kept and changed with the spatial information from the PAN image, respectively.

2. The component of the MS image in the new space that is related or is of similar characteristics with the PAN image is substituted by the high spatial resolution PAN image. In this step, often a histogram matching process is also utilized in an effort to equalization mean and standard deviation for reducing the spectral distortion for both images.

3. The MS image with the substituted PAN image information is transformed back into its original space, resulting in a pan-sharpened image.

CS approach is commonly simplified into the equations below, which basically mean that the PAN image is added to the MS image while the intensity or spatial information derived from the MS image is removed. This addition and removal is multiplied by a coefficient that is determined by the transformation utilized in the process [13].

$$
\begin{aligned}
& \widehat{M S}_{k}=\widetilde{M S}_{k}+g_{k}\left(P-I_{L}\right), \quad k=1, \ldots, N \\
& I_{L}=\sum_{k=1} \omega_{k} \widetilde{M S}{ }_{k}
\end{aligned}
$$

where $\overrightarrow{M S_{k}}$ denotes the pan-sharpened image, $\mathrm{N}$ is the number of bands, $\widetilde{M S}_{k}$ denotes the MS image upsampled to the resolution of PAN image, $P$ is the PAN image and $I_{L}$ is the image synthesized from the MS image bands with weight factors, and $g_{k}$ is a gain factor determined by the utilized transformation in the method [13].

Easy implementation and fast computational time are the major advantages in CS based approaches. In these techniques, if the correlation is very high between PAN and MS images, then the pansharpened MS image has better spatial resolution. The main shortcoming of CS based techniques is the spectral distortion which is also called color or radiometric distortion [14]. 
IHS is one of the standard pansharpening algorithms in the CS category. Pan-sharpening by IHS involves utilizing the IHS transformation to derive the intensity component of the MS image. This intensity component is substituted by the PAN image that is histogram matched to the intensity component. An inverse IHS transformation is used to obtain the pan-sharpened MS image [15].

PCA based pan-sharpening involves utilizing the PCA transformation on the MS image. The first principal component is assumed to be the component with the most spatial information. The PAN image is histogram matched to the first principal component and substituted prior to inverse PCA transformation [16]. The spatial information from the PAN image substituted to the principal component leads to a pan-sharpened MS image after the inverse PCA transformation.

Gram-Schmidt (GS) based pan-sharpening is another standard pan-sharpening approach with utilizes CS. GS transformation results in vectors which are orthogonal with respect to each other. However, the selection of the initial vector, i.e. image, is crucial, and GS based pan-sharpening has several modes to address this issue. In Mode-1, this initial image is formed by taking the average of the MS bands, whereas in Mode- 2 the image is formed by the low-pass filtering of the PAN image and in Mode-3 the minimum least square approach is used [17]. GS based pan-sharpening involves applying GS transformation on the MS image in order to obtain the orthogonal vectors, substituting the histogram matched PAN image in place of the initial vector and using inverse GS process to obtain the pan-sharpened image. GS fusion process can also expressed in the form of equation (1) using the injection gain factor calculated as follows [5]:

$g_{k}=\frac{\operatorname{cov}\left(\widetilde{M S}_{k}, I_{L}\right)}{\operatorname{var}\left(I_{L}\right)}, k=1, \ldots, N$

where $\operatorname{cov}($.$) emphasize the covariance matrix and var(.) de-$ notes the variance value.

In HCS pansharpening approach, MS image is transformed into $\mathrm{n}$-dimensional color space. The squares of the intensity component of the MS image in the color space and the PAN image evaluated. $P^{2}$ is then histogram matched to $I^{2}$ using the means and standard deviations of $P^{2}$ and $P^{2}$. The square root of the histogram matched $P^{2}$ is used in reverse HCS transformation in order to obtain the pan-sharpened image [18].

The main idea in HPF based pan-sharpening is to extract the high frequency details from the PAN image and then inject these details to the MS bands. In the CS based HPF pansharpening, the high frequency details obtained from the PAN image by high-pass filtering are added to each of the MS bands in order to obtain the pan-sharpened image [19].

\section{Modulation-Based (MB) Methods}

In modulation-based pan-sharpening approaches, the ratio of the PAN image and the synthetic PAN image obtained from the
MS image is utilized. This normalized term is multiplied by the MS image bands in order to obtain the pan-sharpened image, as seen in the following equation [20]:

$$
\widehat{M S}_{k}=\left(\frac{\widetilde{M S}_{k}}{S y n}\right) \times P \quad, k=1, \ldots, N
$$

where $\widehat{M S}_{k}$ emphasizes the pan-sharpened image, $\widetilde{M S}_{k}$ denotes the MS image upsampled to the resolution of PAN image, $\mathrm{P}$ denotes the PAN image and the synthetic image (Syn) is obtained from the MS bands, often by a weighted summation such as:

$$
\text { Syn }=\sum_{k=1}^{N} \varphi_{k} \widetilde{M S}
$$

where $\varphi_{k}$ is a weight factor, determined according to the utilized pansharpening method.

It should be noted that some reviews opt out the use of $M B$ group, and instead include the methods presented here in CS or MRA groups, based on some transformations. If such as classification is preferred, BT and UNB methods may be included in the CS group, whereas SFIM and SSCN methods may be included in the MRA group.

Brovey Transformation (BT) is one of the basic pan-sharpening approaches in the modulation-based category. In BT pan-sharpening, the average of the MS bands is taken as the synthetic PAN image in equation (5). This synthetic image is used to normalize the PAN image, and the resulting term is multiplied by each MS band in order to obtain the pan-sharpened image [21].

Smoothing Filter based Intensity Modulation (SFIM) is a modulation based pansharpening technique which assumes that the radiance ratio between high spatial resolution MS band and original PAN image is equal to the resolution ratio between original MS band and low-pass filtered PAN image. The synthetic image is derived by using a kernel whose size is based on this resolution ratio. The high resolution details, obtained by the normalization of the PAN image with its low-pass synthetic version, is multiplied with the original MS bands in order to create the pansharpened image [22].

University of New Brunswick (UNB) approach utilizes least squares regression between the MS and PAN images in order to find the weight factors. These weights are multiplied by each MS band to obtain the synthetic PAN image. In order to create the pansharpened image, each MS band is multiplied by the ratio between the original PAN and the obtained synthetic PAN image [23]. In this work, Fully Constraint Least Squares (FCLS) algorithm is utilized for the regression analysis.

Spectral Simulation Color Normalization (SSCN) based pansharpening utilizes the concept of sensor spectral response functions (SRF). By using the FCLS regression analysis, the 
weights to approximate the PAN SRF from the MS SRFs are calculated, and then the simulated PAN image is obtained by using these weights. Each MS band is then multiplied by the ratio between the PAN image and the simulated PAN image which is histogram matched with PAN, in order to obtain the pan-sharpened image [24].

\section{Multi-Resolution Analysis (MRA)}

In order to overcome the spectral distortion shortcoming of the CS approach, MRA methods have been developed. In MRA methods, MS and PAN images are first decomposed into frequency bands. Then, the details coming from high frequency component of the PAN image are injected into the frequency bands of the MS image which have been interpolated to the PAN resolution. In this way, MRA based approaches preserve spectral information better than CS methods. MRA approach is obtained as follows [5]:

$$
\widehat{M S}_{k}=\widetilde{M S}_{k}+G_{k} \otimes\left(P-P_{L}\right) \quad, \quad k=1, \ldots, N
$$

where $\widehat{M S}_{k}$ means the pan-sharpened image, $\widetilde{M S}_{k}$ denotes the MS image upsampled to the resolution of PAN image, $\otimes$ denotes element-wise multiplication, $P$ is the PAN image and $P_{L}$ denotes that low pass version of the PAN image $P$.

The most popular MRA approaches are the wavelet transformation and Laplacian pyramids based approaches. Both of those groups can be represented in a pyramidal scheme as in [2], and are often commonly termed as the ARSIS concept. ARSIS concept is motivated by obtaining the missing spatial information of the MS image from the high frequencies of PAN image between level-0 and level-1 on the pyramid.

MRA based pansharpening techniques have the advantages of temporal cohorence, spectral consistency and robustness to aliasing, whereas the complexity and higher computation time with respect to CS methods can be counted as the disadvantages of MRA [25].

Wavelet Transformation (WT) is the name of a family of methods used to decompose an image into its frequency components based on the utilized basis function. This decomposition is commonly conducted into four components, the first of which is the approximation component, the second and third components are the horizontal and vertical details and the fourth includes the frequency components that are both horizontal and vertical.

Wavelet Transformation based pan-sharpening starts with the histogram matching of the PAN image with each of the MS bands. For each MS band, WT is applied to the histogram matched PAN image, and the second, third and fourth components are set to zero, resulting in only the approximation component remaining. Inverse WT is applied to this component, and the result is subtracted from the original PAN image in order to obtain the high frequency details. These details are added to the respective MS bands, and the pan-sharpened image is obtained [26].
In the literature, instead of the regular discrete WT, stationary WT, more commonly named as A Tróus WT (ATWT) is preferred because of the enhanced pansharpening performance it provides. In this work, for ATWT, the following cubic-spline kernel developed by Starck and Murtagh is utilized [27].

$$
h=\frac{1}{256}\left[\begin{array}{ccccc}
1 & 4 & 6 & 4 & 1 \\
4 & 16 & 24 & 16 & 4 \\
6 & 24 & 36 & 24 & 6 \\
4 & 16 & 24 & 16 & 4 \\
1 & 4 & 6 & 4 & 1
\end{array}\right]
$$

Laplacian Pyramids (LP), which was first introduced by Burt and Adelson, is derived from Gaussian Pyramid (GP) which utilizes low-pass filter recursively by using reduction and decimation [28]. In GP, the first level is taken as original image, and each upper level image is obtained by low pass filtered lower level image, sequentially. In LP, each level includes a detail image which is obtained by the subtraction of the interpolated lower level image from the same level image. Hence, while GP can be considered as a set of images which constitutes the low-pass filtered versions of an original image, LP can be seen as a set of image details obtained as the difference between the lower level images and their band-pass filtered versions. LP based pansharpening is motivated by extracting high spatial details from the PAN image by the use of LPs, and adding these details to the MS bands.

Enhanced Laplacian Pyramid (ELP) is an MRA technique in which the successive levels of the pyramid should have the lower correlation and zeroth level entropy properties [29]. In ELP, unlike in LP, it is possible to construct different types of kernels for the expansion and reduction operations. Generalized Laplacian Pyramid (GLP) is generalized from ELP by enabling the use fractional numbers as scale ratios. GLP approach is reported to be more advantageous than A Tróus Wavelet transform [30]. In the literature, GLP has also been recently adopted as the name of all LP based pan-sharpening approaches.

Taking into consideration the frequency response functions of different kernels, 23tap filter is suggested in the literature for constructing the LP, i.e. for the expansion of the lower level images, for the higher performances it provides [31], and is also adopted in this work.

Modulation transfer function (MTF) is the amplitude spectrum of the system point spread function. MTFs of the imaging system are different from each other for both MS and PAN images. Generalized Laplacian Pyramid with MTF-matched filter (MTFGLP) is another MRA based technique. In MTF-GLP pan-sharpening approach, MTFs of the imaging sensor are exploited to design the GLP reduction filter. After the PAN image is reduced by using the MTF filter, interpolation is performed on the histogram matched PAN image by utilizing a 23-tap kernel. Then, the detail image is calculated by subtracting the obtained low resolution PAN image from the original PAN image. Finally, these details are added into the original MS bands to obtain the pan-sharpened image [32]. 
MTF-GLP-HPM pan-sharpening approach follows the steps of MTF-GLP pan-sharpening, but in the last step high pass modulation injection method is employed in order to obtain the pan-sharpened image [33].

\section{Hybrid Methods}

Hybrid pansharpening approaches are a cross between the different pansharpening approach groups and do not belong to a single one. Among such methods, Ehlers fusion, guided filter PCA (GFPCA), and IHS based wavelet transform may be counted among others [34]. In this work, Ehlers fusion is included as a hybrid pansharpening method. Ehlers fusion uses IHS transformation and filtering in the Fourier domain. For the first step, MS bands are transformed to IHS domain. The intensity component and the PAN image is transformed into Fourier domain for filtering. After the low-pass filtering of the intensity component and the high pass filtering of the PAN image, inverse Fourier transform is applied to the filter outputs. Filtered PAN image is added to the filtered intensity component in the IHS domain and inverse IHS transformation is applied in order to obtain the pansharpened image [35].

\section{Variational Approaches}

$\mathrm{P}+\mathrm{XS}$ is a variational pansharpening technique which aims to minimize an energy function by using the Gradient Descent algorithm. The energy function utilized in P+XS is motivated by the assumption that the geometry of the spectral bands are contained in the topographic map of the PAN image and the relationship between the MS bands and the PAN image. P+XS also assumes a low-pass filtering and subsampling approximation relationship between the MS bands and the PAN image in order to construct the energy function [36]. The energy function involves three terms to enforce and utilize these assumptions and is optimized by gradient descent in order to obtain the pan-sharpened image. The derivation of the energy function and its terms are explained in detail in [36].

\section{Experimental Results}

\section{Datasets}

The datasets used in this study are captured by RASAT remote sensing satellite from different regions of Turkey. The datasets are downloaded from the GEZGiN geoportal, and subsets of the downloaded images are used for computational purposes. MS images are taken as $150 \times 150$ pixels, and have three bands, whereas the PAN images have $300 \times 300$ pixels coinciding with the same region as in the MS images. The pixel size difference is caused by the resolution ratio as the spatial resolution of the MS images are $15 \mathrm{~m}$, whereas the spatial resolution of the PAN images are $7.5 \mathrm{~m}$.

Experimental results are provided in this work for three pairs of datasets. The datasets were carefully selected based on the properties of their captured scenes. The first image pair are captured on 15.06.2015 from Mersin region, and contain extensive farm fields, which results in homogeneous patches and unpronounced edges. The second image pair are captured on 12.01.2014 from İstanbul region, and comprise of high spatial frequency containing urban areas, and coast-to-land transitions. The third image pair are captured on 31.08.2015 from
Sin- region, and contain roads, land-cover and sea surfaces. The color and grayscale visuals for the MS and PAN images, respectively, are provided below in Figure 1-3.

\section{Wald's protocol}

In order to evaluate the performance of pan-sharpening methods quantitatively, a reference image is required to compare with the resulting pan-sharpened images. To overcome this shortcoming, various approaches have been proposed in the literature for performance comparison purposes. The most widely accepted and utilized of these approaches is the Wald's protocol [37]. In Wald's

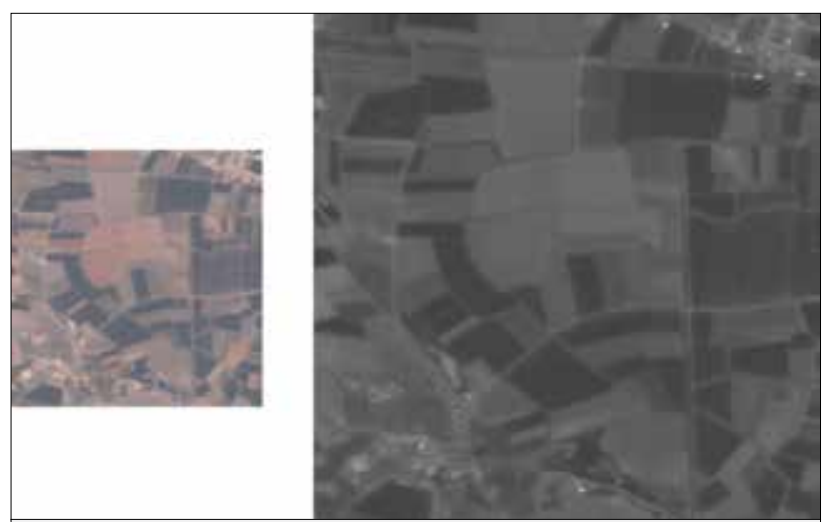

Figure 1. RGB and PAN images for the Mersin dataset



Figure 2. RGB and PAN images for the İstanbul dataset

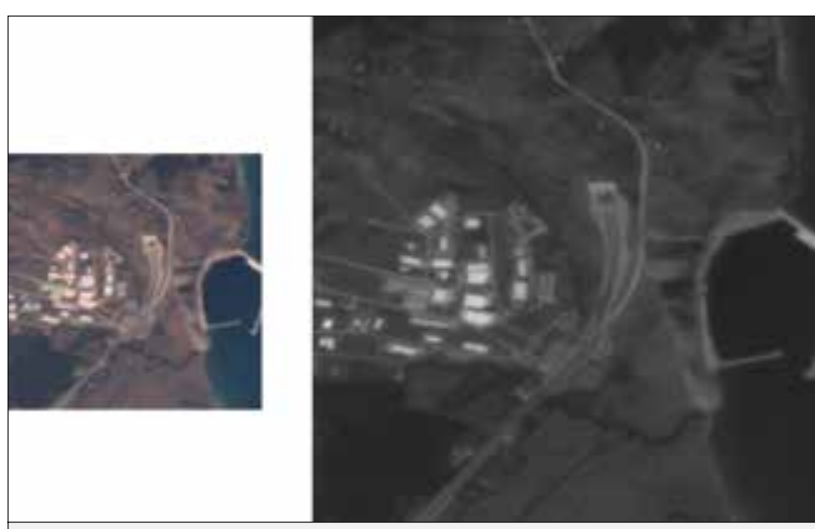

Figure 3. RGB and PAN images for the Sinop dataset 
protocol, which is also adopted in this work, the spatial resolution of the MS and PAN images are first reduced, and pan-sharpening methods are applied on these reduced resolution images. The resulting pan-sharpened image is compared with the original MS image to evaluate the pan-sharpening performance.

\section{Results and Discussion}

Experimental results obtained in accordance with Wald's protocol are provided in this section. Three datasets have been utilized and the results are provided both visually and quantitatively. For the quantitative results, the performance metrics of RMSE, CC, SAM, ERGAS, PSNR SSIM and UIQI are used. The quantitative results for the three datasets with seven quality assessment metrics and using seventeen pan-sharpening methods are provided in Table 1-3, for the Mersin, İstanbul and Sinop datasets, respectively. The visual results are provided in Figure 4-6, for the Mersin, İstanbul and Sinop datasets, respectively.

It can be observed from the Tables that the state-of-the-art MTF-GLP-HPM method provides the best results overall, followed closely by MTF-GLP, which is in turn followed by ATWT. This is to be expected as utilizing sensor MTFs in the pan-sharpening process enables to obtain better performances than clas- sical approaches [31]. Because of this, whereas ATWT outperforms the basic form of GLP, the inclusion of MTFs to GLP results is significantly better performances.

As can be seen from Figure 4-6, the visual results are also in accord with the quantitative evaluations in that the MTF-GLP-HPM provides the sharpest pan-sharpened images without any color distortion, followed closely in performance with MTF-GLP, ATWT and SFIM. Among the more classical approaches in the CS and MB categories which do not utilize sensor specifications, often blur effects or slight color distortions can be observed in the pan-sharpened visuals. The spatial degradation, i.e. the blurry appearance, is easier to observe on the farm borders in Figure 4, sea-coast border and the small structures in Figure 5, and the main road and the buildings in Figure 6 . This afore mentioned effect is more prevalent in the visual outputs of Ehlers, GLP (without the utilization of MTFs), and GS2 methods.

An important point to note is that all methods performed better in Mersin image than in Sinop image. This is also expected, as the high number of high frequency components in the Sinop image makes it a more challenging dataset with respect to Mersin image.

Table 1. Quantitative pan-sharpening results for Mersin dataset

\begin{tabular}{|c|c|c|c|c|c|c|c|}
\hline & RMSE & CC & SAM & ERGAS & PSNR & SSIM & UIQI \\
\hline IHS & 4.10 & 0.98 & $<0.01$ & 1.51 & 35.0 & 0.97 & 0.97 \\
\hline PCA & 4.07 & 0.98 & $<0.01$ & 1.50 & 35.2 & 0.98 & 0.97 \\
\hline GS1 & 4.06 & 0.98 & $<0.01$ & 1.50 & 35.1 & 0.97 & 0.97 \\
\hline GS2 & 4.76 & 0.97 & $<0.01$ & 1.76 & 33.8 & 0.97 & 0.96 \\
\hline GS3 & 4.07 & 0.98 & $<0.01$ & 1.50 & 35.1 & 0.97 & 0.97 \\
\hline HCS & 4.08 & 0.98 & $<0.01$ & 1.51 & 35.1 & 0.97 & 0.97 \\
\hline HPF & 4.60 & 0.97 & $<0.01$ & 1.70 & 34.0 & 0.97 & 0.96 \\
\hline BT & 3.86 & 0.98 & $<0.01$ & 1.43 & 35.5 & 0.98 & 0.98 \\
\hline SFIM & 3.62 & 0.98 & $<0.01$ & 1.33 & 36.4 & 0.98 & 0.98 \\
\hline UNB & 4.02 & 0.98 & $<0.01$ & 1.49 & 35.1 & 0.97 & 0.97 \\
\hline SSCN & 4.13 & 0.98 & $<0.01$ & 1.53 & 34.9 & 0.97 & 0.97 \\
\hline ATWT & 3.24 & 0.99 & $<0.01$ & 1.20 & 37.1 & 0.99 & 0.98 \\
\hline GLP & 4.28 & 0.98 & $<0.01$ & 1.58 & 34.8 & 0.97 & 0.97 \\
\hline MTF-GLP & 2.73 & 0.99 & $<0.01$ & 1.01 & 38.4 & 0.99 & 0.99 \\
\hline MTF-GLP-HPM & 2.71 & 0.99 & $<0.01$ & 1.01 & 38.5 & 0.99 & 0.99 \\
\hline Ehlers & 4.91 & 0.97 & $<0.01$ & 1.81 & 33.6 & 0.96 & 0.96 \\
\hline PXS & 3.59 & 0.98 & $<0.01$ & 1.33 & 36.2 & 0.98 & 0.98 \\
\hline
\end{tabular}

RMSE: root mean squared error; CC: correlation coefficient; SAM: spectral angular mapper; ERGAS: Erreur Relative Globale Adimensionnelle de Synthese; PSNR: peak signal-to-noise ratio; SSIM: structural similarity index; UIQI: universal image quality index 
Table 2. Quantitative pan-sharpening results for İstanbul dataset

\begin{tabular}{|c|c|c|c|c|c|c|c|}
\hline & RMSE & CC & SAM & ERGAS & PSNR & SSIM & UIQI \\
\hline IHS & 4.10 & 0.96 & $<0.02$ & 2.77 & 34.5 & 0.95 & 0.93 \\
\hline PCA & 4.06 & 0.96 & $<0.02$ & 2.74 & 34.5 & 0.95 & 0.93 \\
\hline GS1 & 4.08 & 0.96 & $<0.02$ & 2.75 & 34.5 & 0.95 & 0.93 \\
\hline GS2 & 4.57 & 0.95 & $<0.02$ & 3.08 & 33.5 & 0.93 & 0.91 \\
\hline GS3 & 4.07 & 0.96 & $<0.02$ & 2.75 & 34.5 & 0.95 & 0.93 \\
\hline $\mathrm{HCS}$ & 4.21 & 0.96 & $<0.02$ & 2.84 & 34.3 & 0.95 & 0.93 \\
\hline HPF & 4.31 & 0.95 & $<0.02$ & 2.91 & 34.1 & 0.95 & 0.93 \\
\hline BT & 3.89 & 0.96 & $<0.02$ & 2.62 & 34.9 & 0.96 & 0.95 \\
\hline SFIM & 3.69 & 0.97 & $<0.02$ & 2.50 & 35.6 & 0.96 & 0.95 \\
\hline UNB & 4.07 & 0.96 & $<0.02$ & 2.74 & 34.5 & 0.95 & 0.93 \\
\hline SSCN & 4.12 & 0.96 & $<0.02$ & 2.77 & 34.4 & 0.95 & 0.93 \\
\hline ATWT & 3.41 & 0.97 & $<0.02$ & 2.30 & 36.1 & 0.97 & 0.96 \\
\hline GLP & 4.48 & 0.95 & $<0.02$ & 3.02 & 33.7 & 0.94 & 0.92 \\
\hline MTF-GLP & 3.12 & 0.97 & $<0.01$ & 2.10 & 36.8 & 0.97 & 0.96 \\
\hline MTF-GLP-HPM & 3.10 & 0.98 & $<0.01$ & 2.08 & 36.8 & 0.97 & 0.96 \\
\hline Ehlers & 5.02 & 0.94 & $<0.02$ & 3.39 & 32.7 & 0.92 & 0.90 \\
\hline PXS & 3.74 & 0.96 & $<0.02$ & 2.52 & 35.3 & 0.96 & 0.95 \\
\hline
\end{tabular}

RMSE: root mean squared error; CC: correlation coefficient; SAM: spectral angular mapper; ERGAS: Erreur Relative Globale Adimensionnelle de Synthese; PSNR: peak signal-to-noise ratio; SSIM: structural similarity index; UIQI: universal image quality index

Table 3. Quantitative pan-sharpening results for Sinop dataset

\begin{tabular}{|c|c|c|c|c|c|c|c|}
\hline & RMSE & CC & SAM & ERGAS & PSNR & SSIM & UIQI \\
\hline IHS & 5.92 & 0.98 & $<0.01$ & 3.18 & 32.4 & 0.97 & 0.97 \\
\hline PCA & 6.13 & 0.98 & $<0.02$ & 3.29 & 32.2 & 0.97 & 0.97 \\
\hline GS1 & 5.97 & 0.98 & $<0.01$ & 3.20 & 32.4 & 0.97 & 0.97 \\
\hline GS2 & 6.14 & 0.98 & $<0.01$ & 3.29 & 32.1 & 0.97 & 0.97 \\
\hline GS3 & 6.22 & 0.98 & $<0.02$ & 3.33 & 32.1 & 0.97 & 0.97 \\
\hline HCS & 7.05 & 0.98 & $<0.01$ & 3.77 & 30.8 & 0.96 & 0.95 \\
\hline HPF & 5.27 & 0.98 & $<0.01$ & 2.82 & 33.5 & 0.98 & 0.98 \\
\hline BT & 5.67 & 0.98 & $<0.01$ & 3.04 & 32.8 & 0.98 & 0.98 \\
\hline SFIM & 4.56 & 0.99 & $<0.01$ & 2.44 & 34.9 & 0.99 & 0.98 \\
\hline UNB & 5.81 & 0.98 & $<0.01$ & 3.11 & 32.5 & 0.97 & 0.97 \\
\hline SSCN & 6.51 & 0.97 & $<0.01$ & 3.48 & 31.5 & 0.97 & 0.96 \\
\hline ATWT & 4.37 & 0.99 & $<0.01$ & 2.34 & 35.3 & 0.99 & 0.99 \\
\hline GLP & 6.09 & 0.98 & $<0.01$ & 3.26 & 32.2 & 0.97 & 0.97 \\
\hline MTF-GLP & 3.98 & 0.99 & $<0.01$ & 2.14 & 36.0 & 0.99 & 0.99 \\
\hline MTF-GLP-HPM & 3.91 & 0.99 & $<0.01$ & 2.10 & 36.2 & 0.99 & 0.99 \\
\hline Ehlers & 6.77 & 0.97 & $<0.01$ & 3.63 & 31.3 & 0.96 & 0.96 \\
\hline PXS & 5.02 & 0.98 & $<0.01$ & 2.69 & 33.9 & 0.98 & 0.98 \\
\hline
\end{tabular}

RMSE: root mean squared error; CC: correlation coefficient; SAM: spectral angular mapper; ERGAS: Erreur Relative Globale Adimensionnelle de Synthese; PSNR: peak signal-to-noise ratio; SSIM: structural similarity index; UIQI: universal image quality index 


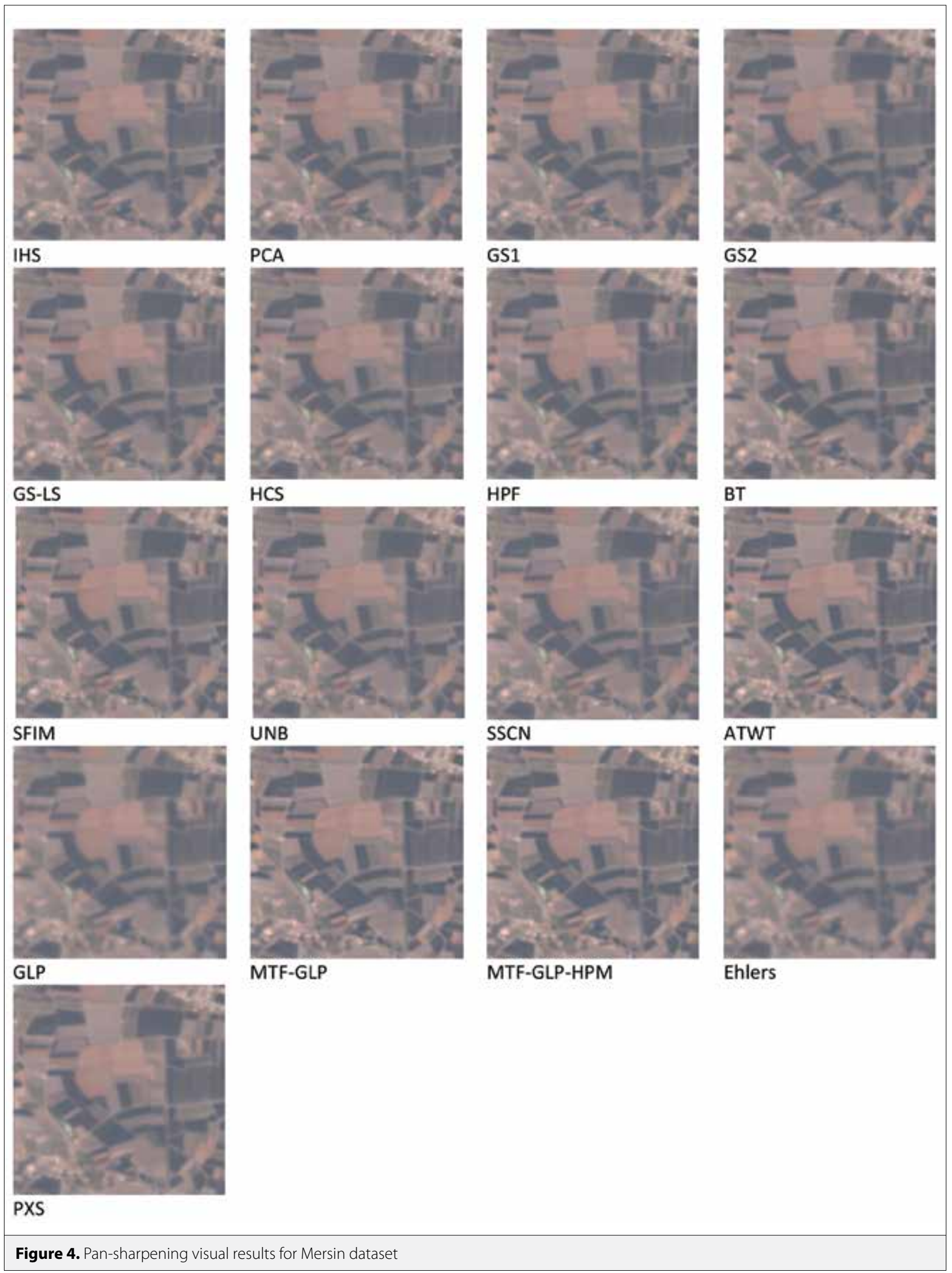




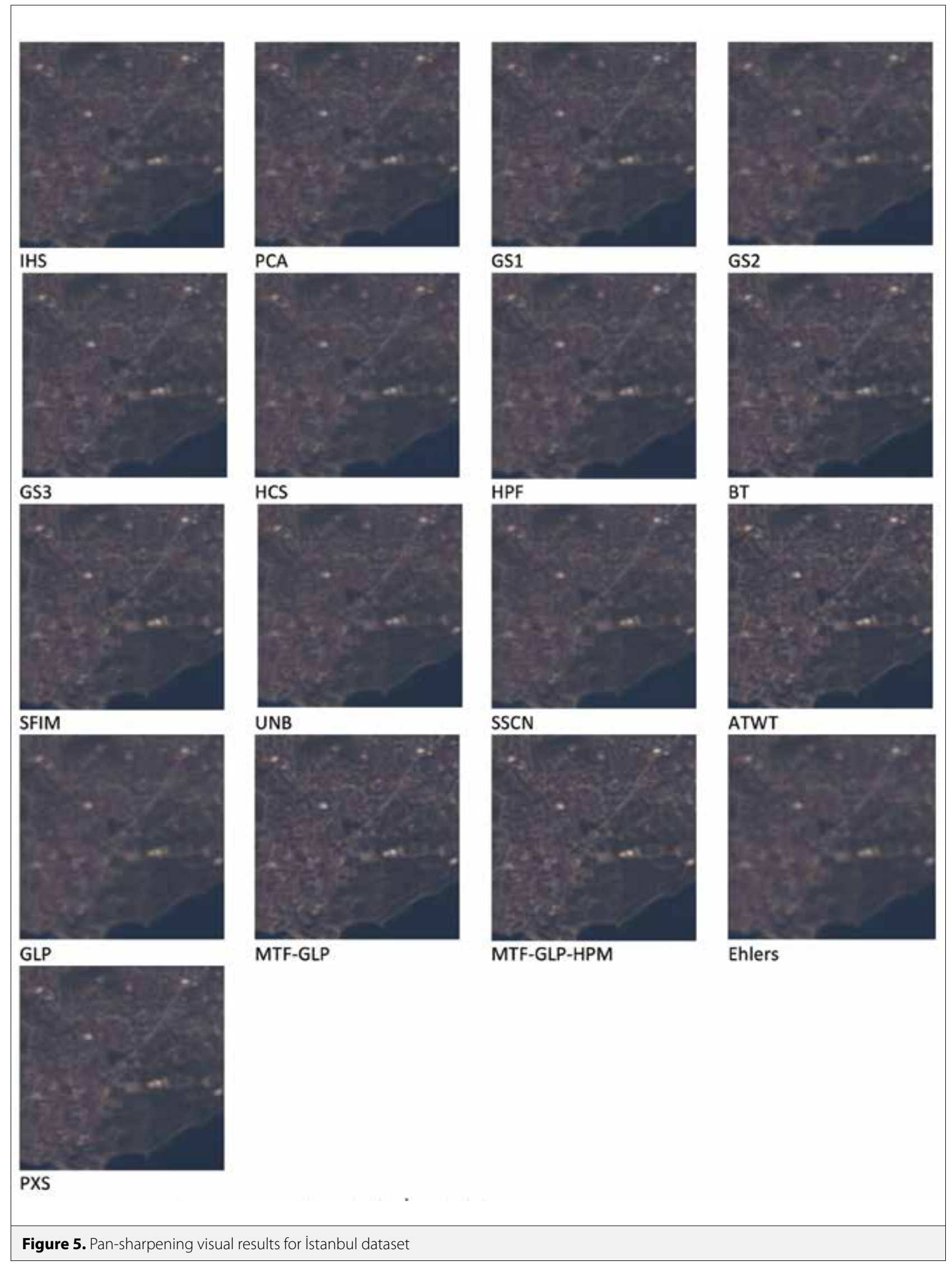




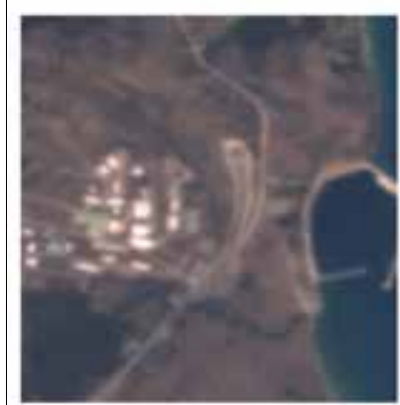

IHS

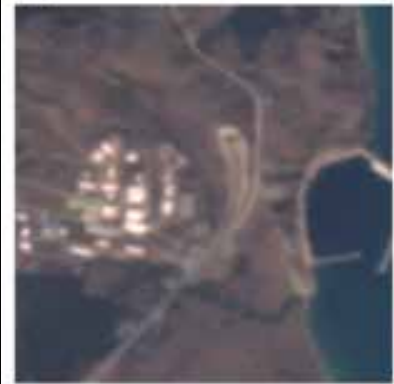

GS3

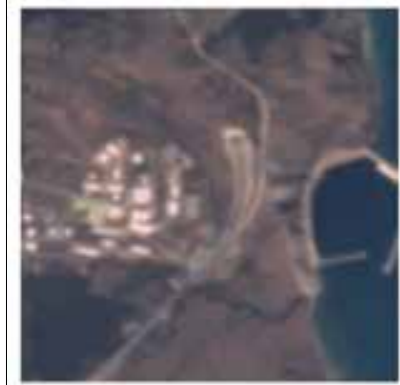

SFIM

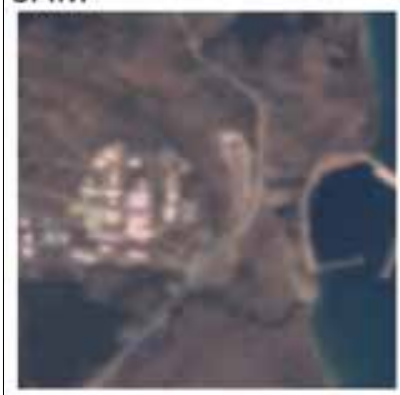

GLP

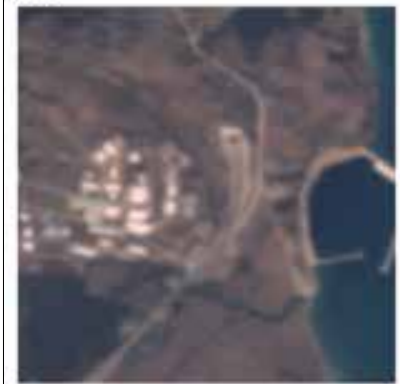

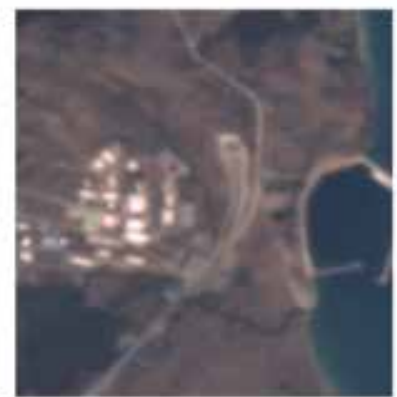

PCA

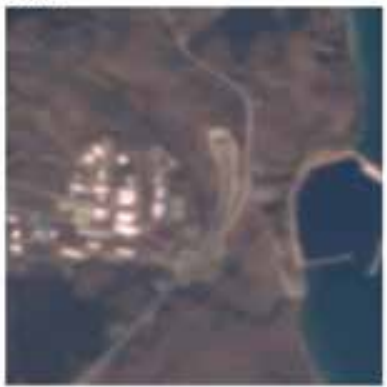

HCS

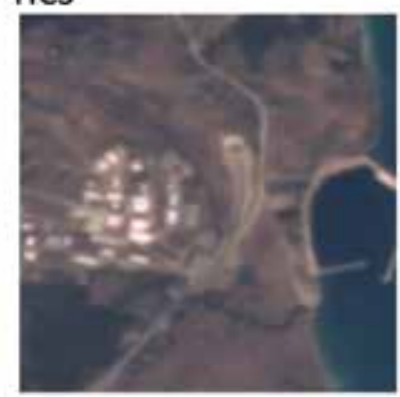

UNB

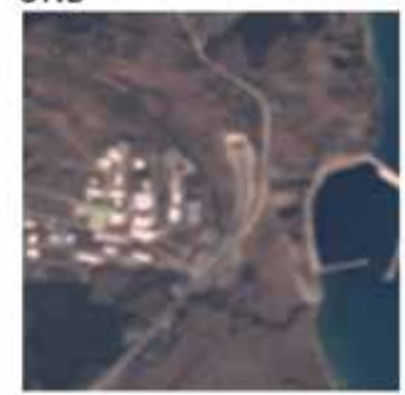

MTF-GLP

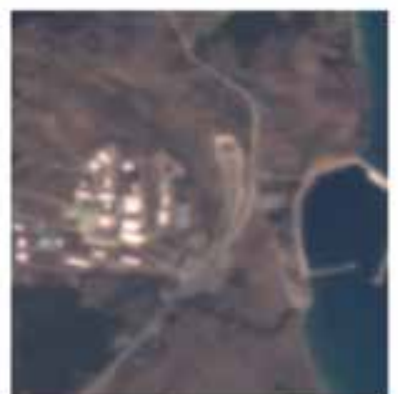

GS1

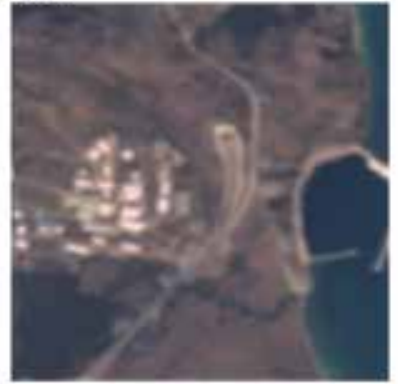

HPF

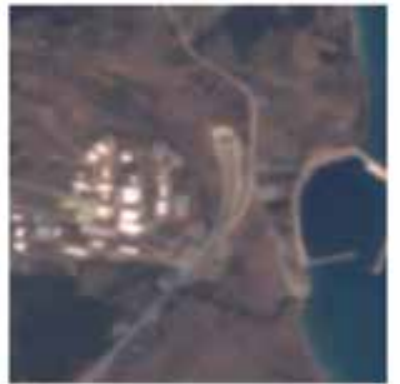

SSCN

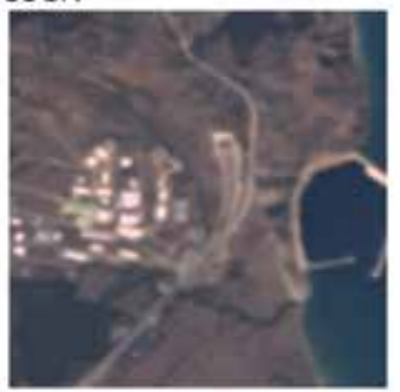

MTF-GLP-HPM

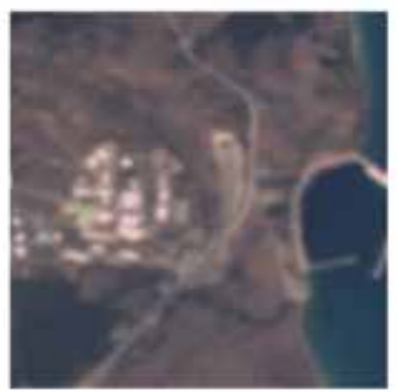

GS2

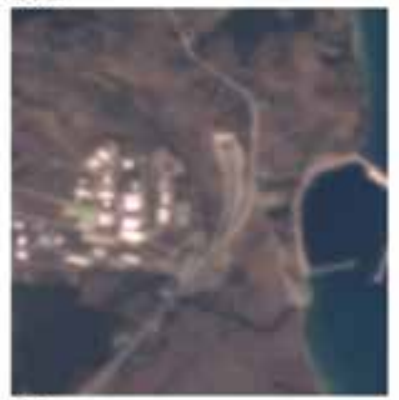

BT

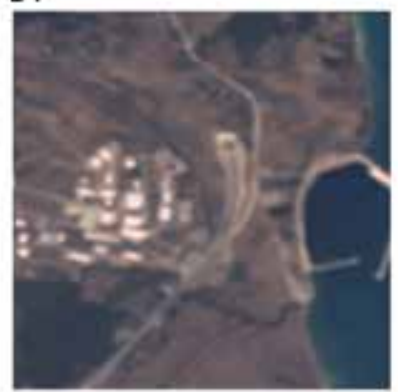

ATWT

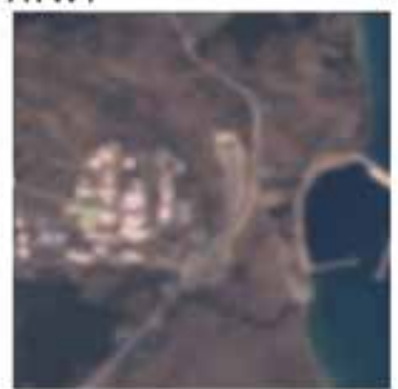

Ehlers

PXS

Figure 6. Pan-sharpening visual results for Sinop dataset 
In [7], it is mentioned that HPF and HCS approaches give the best pansharpening performance results among the other utilized methods in [7], which is also in accordance with the results of this work. In [10], it is stated that PCA provides the best performance quantitatively, but BT provides the best visual result for RASAT satellite images. However, in our experiments BT showed better performance with respect to IHS and PCA approaches both qualitatively and quantitatively. In [5], state-of-the-art pansharpening approaches were studied and applied to IKONOS-2 and WorldView-2 satellites, and the MTF-GLP-HPM approach provided the best performance, as it also did in this study.

\section{Conclusions}

The aim of this paper was to provide the most comprehensive review and performance comparison of pansharpening methods for RASAT images up-to-date. The experimental validation procedure followed Wald's protocol in accordance with current literature. Experimental results were provided for three datasets of different properties due to their scenes.

It was determined through both qualitative and quantitative analysis that MTF-GLP-HPM provides the best performance overall among all groups of pan-sharpening approach, whether CS, MB, MRA, hybrid or variational. This points out that utilizing the MTFs of the sensor provides a significant boost in the pan-sharpening performance.

It was also observed that overall, the MRA based pansharpening methods provide enhanced performance with respect to CS or MB based methods. Whereas there was no clear best performing method in the CS group, SFIM outperformed the other methods in the MB group. The variational $\mathrm{P}+\mathrm{XS}$ method is seen to be competing with SFIM in terms of performance, whereas the hybrid Ehlers method is outperformed by many of the utilized methods.

Future studies may include a similar review of pan-sharpening methods' performance for GÖKTÜRK-2 images, which also contain the near infrared spectral band, or adapting the various methodologies for the fusion of different sensor images, such as hyperspectral - multispectral fusion.

\section{Acknowledgments}

This work is supported by TÜBITAK under project no $115 \mathrm{E} 883$. The authors would like to thank TÜBITAK Uzay for making the RASAT images publicly available, and for providing the SRFs and MTFs for the RASAT sensor.

\section{References}

1. A. Garzelli, F. Nencini, L. Alparone, B. Aiazzi, and S. Baronti, "Pan-sharpening of multispectral images: a critical review and comparison," IGARSS IEEE International Geoscience and Remote Sensing Symposium, vol.1, pp.81-84, 2004. [CrossRef]
2. C. Thomas, T. Ranchin, L Wald and J. Chanussot, "Synthesis of multispectral images to high spatial resolution: A critical review of fusion methods based on bemote sensing physics," IEEE Transactions on Geoscience and Remote Sensing, vol. 46, pp. 1301 - 1312, 2008. [CrossRef]

3. Y. Zhang and. R. K. Mishra, "A review and comparison of commercially available pan-sharpening techniques for high resolution satellite image fusion," IEEE International Geoscience and Remote Sensing Symposium, pp. 182-185, 2012.

4. J. Marcello, A. Medina F. Eugenio, "Evaluation of spatial and spectral effectiveness of pixel-level fusion techniques," IEEE Geoscience and Remote Sensing Letters, vol. 10, no. 3, pp. 432-436, 2013. [CrossRef]

5. G. Vivone, L. Alparone, J. Chanussot, M. D. Mura, A. Garzelli, L. G. A. Licciardi, R. Restaino, L. Wald, "A critical comparison among pansharpening algorithms," IEEE Transactions on Geoscience and Remote Sensing, vol. 53, no.5, pp. 2565-2586, 2015. [CrossRef]

6. L. Loncan, L. B. de Almeida, J. M. Bioucas-Dias, X. Briottet, J. Chanussot, N. Dobigeon, S. Fabre, L. W. Liao, G. A. Licciardi, M. Simoes, T. J.Y. Tourneret, M. A. Veganzones, G. Vivone, Q. Wei and N. Yokoya, "Hyperspectral pansharpening: A review," IEEE Geoscience and Remote Sensing Magazine, vol. 3, no. 3, pp. 27-46, 2015. [CrossRef]

7. I. Amro, J. Mateos, M. Vega, R. Molina, A. K. Katsaggelos, "A survey of classical methods and new trends in pansharpening of multispectral images," Journal on Advances in Signal Processing EURASIP, vol. 79, 2011.

8. A. D. Vaiopoulos, K. Karantzalos, "Pansharpening on the narrow VNIR and SWIR spectral bands of Sentinel-2", The International Archives of the Photogrammetry, Remote Sensing and Spatial Information Sciences, XLI-B7, XXIII ISPRS Congress, 2016. [CrossRef]

9. M. Teke, M. S. Seyfioğlu, A. Ağçal, S. Z. Gürbüz, "RASAT Uydu görüntülerinin optimal pankeskinleştirilmesi", IEEE 22. Sinyal İsleme ve iletişim Uygulamaları Kurultayı (SIU), pp. 1967-1970, 2014.

10. I. S. Açıkgöz, S., T. M. Teke, K. U. Kutbay, F. Hardalaç, "Performance evaluation of pansharpening methods on GPU for RASAT images", 7th International Conference on Recent Advances in Space Technologies (RAST), pp. 283-288, 2015.

11. M. Teke, "Satellite image processing workflow for RASAT and Göktürk-2," Journal of Aeronautics and Space Technologies, vol. 9 no. 1 pp. 1-13, 2016.

12. M. Özendi, H. Topan, A. Cam and Ç. Bayık, "RASAT ve GÖKTÜRK-2 görüntülerinin pankeskinleştirilmiş görüntü üretimi ve kalite değerlendirilmesi," 6. Uzaktan Algılama-CBS Sempozyumu (UZALCBS 2016), pp. 447-453, 2016.

13. W. Dou, Y. Chen, X. Li, D. Z. Sui, "A general framework for component substitution image fusion: An implementation using the fast image fusion method", Computational Geoscience, vol. 33 no.2, pp. 219-228, 2007. [CrossRef]

14. B. Aiazzi, S. Baronti, M. Selva, "Improving Component Substitution Pansharpening through multivariate regression of MS+Pan data," IEEE Transactions on Geoscience and Remote Sensing, vol. 45, no. 10, pp. 3230-3239, 2007. [CrossRef]

15. T. M. Tu, S. C. Su, S. H. C. Shyu, P. S. Huang, "A new look at IHS-like image fusion methods," Information Fusion, vol. 2, no. 3, pp. 177186, 2001. [CrossRef]

16. P. S. Chavez, Jr. S. C. Sides, J. A. Anderson, “Comparison of three different methods to merge multiresolution and multispectral data: Landsat TM and SPOT panchromatic," Photogramm. Eng. Remote Sens., vol. 57, no. 3, pp. 295-303, 1991. 
17. C. A. Laben and B. V. Brower, "Process for enhancing the spatial resolution of multispectral imagery using pan-sharpening," U.S. Patent 6011 875, 2000.

18. C. Padwick, M. Deskevich, F. Pacifici, S. Smallwood, "WorldView-2 pan-sharpening", ASPRS Annual Conference, 2010.

19. G. U. G. Gangkofner, P. S. Pradhan, D. W. Holcomb, "Optimizing the high-pass filter addition technique for image fusion," Photogramm. Eng. Remote Sens., vol. 74, no. 9, pp.1107-1118, 2008. [CrossRef]

20. Y. Junghui, Z. Jixian, L. Haitao, S. Yushan, P. Pengxian, “Pixel level fusion methods for remote sensing images: A current review", ISPRS TC VII Symposium, vol. 38, pp. 680-686, Vienna, Austria, 2010.

21. A. R. Gillespie, A. B. Kahle, R. E. Walker, "Color enhancement of highly correlated images-II. Channel ratio and "chromaticity" transform techniques," Remote Sensing Env., vol. 22, no. 3, pp. 343365, 1987. [CrossRef]

22. J. G. Liu, "Smoothing filter-based intensity modulation: A spectral preserve image fusion technique for improving spatial details," International Journal of Remote Sensing, vol. 21, no.18, pp. 34613472, 2000. [CrossRef]

23. Y. Zhang, "A new merging method and its spectral and spatial effects," International Journal of Remote Sensing, vol. 20, no. 10, pp. 2003-2014, 1999. [CrossRef]

24. N. Yokoya, N. Mayumi, A. Iwasaki, "Cross-calibration for data fusion of EO-1 / Hyperion and Terra / ASTER," IEEE Journal of Selected Topics in Applied Earth Observations and Remote Sensing, vol. 6, no. 2, pp. 419-426, 2013. [CrossRef]

25. S. Baronti, B. Aiazzi, M. Selva, A. Garzelli, L. Alparone, "A theoretical analysis of the effects of aliasing and misregistration on pansharpened imagery," IEEE Journal of Selected Topics in Signal Processing, vol. 5, no. 3, pp. 446-453, 2011. [CrossRef]

26. F. Murtagh, S. J.L. Starck, A. Bijaoui, "Image restoration with noise suppression using a multiresolution support," Astronomy and Astrophysics supplement series, vol. 112, pp.179-189, 1995.

27. J. Nunez, X. Otazu, O. Fors, A. Prades, V. Pala, R. Arbiol, "Multiresolution-based image fusion with additive wavelet decomposition,"
IEEE Transactions on Geoscience and Remote Sensing, vol. 37, no. 3, pp. 1204-1211, 1999. [CrossRef]

28. P. Burt and E. Adelson, "The Laplacian Pyramid as compact image code," IEEE Transactions on Communications, vol. 31, no. 4, pp. 532 - 540, 1983. [CrossRef]

29. S. Baronti, A. Casini, F. Lotti, L. Alparone, "Context-driven differential encoding of an enhanced image pyramid," Signal Processing Image Communication, vol. 6, pp. 463-469, 1994. [CrossRef]

30. B. Aiazzi, S. Baronti, L. Alparone, A. Garzelli, M. Selva, "Advantages of Laplacian pyramids over "à trous" wavelet transforms for pansharpening of multispectral images," Proceedings of SPIE-The International Society for Optical Engineering, 2012. [CrossRef]

31. B. Aiazzi, A. L. Alparone, S. Baronti, A. Garzelli, "Context-driven fusion of high spatial and spectral resolution images based on oversampled multiresolution analysis," IEEE Transactions on Geoscience and Remote Sensing, vol. 40, no. 10, pp. 2300-2312, 2002. [CrossRef]

32. B. Aiazzi, L. Alparone, S. Baronti, A. Garzelli, M. Selva, "MTF-tailored multiscale fusion of high resolution MS and Pan imagery," Photogramm. Eng. Remote Sens., vol. 72, no. 5, pp. 591-596, 2006. [CrossRef]

33. J. Lee and C. Lee, "Fast and efficient panchromatic sharpening," IEEE Trans. Geosci. Remote Sens., vol. 48, no. 1, pp. 155-163, Jan. 2010. [CrossRef]

34. Y. Chibani and A. Houacine, "The joint use of IHS transform and redundant wavelet decomposition for fusing multispectral and panchromatic images," International Journal of Remote Sensing, vol. 23, no. 18, pp. 3821-3833, 2002. [CrossRef]

35. S. Klonus and M. Ehlers, "Image fusion using the Ehlers spectral characteristics preservation algorithm," GIScience \& Remote Sensing, vol. 44, no.2, pp. 93-116, 2007. [CrossRef]

36. C. Ballester, V. Caselles, L. Igual, J. Verdera, B. Rougé, "A variational model for $\mathrm{P}+\mathrm{XS}$ image fusion", International Journal of Computer Vision, vol. 69, no. 1, pp. 43-58, 2006. [CrossRef]

37. L. Alparone, B. Aiazzi, S. Baronti, A. Garzelli, F. Nencini and M. Selva, "Multispectral and panchromatic data fusion assessment without reference," Photogrammetric Engineering \& Remote Sensing, vol. 74, no. 2, pp. 193-200, Feb. 2008. [CrossRef]

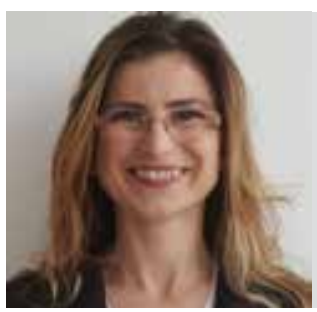

Sevcan Kahraman received the B.Sc. degree in Mathematics department at Dokuz Eylül University in 2005, and the M.Sc. degree in Mathematics at Izmir Institute of Technology in 2008. She is currently a Ph.D student and working at the Kocaeli University Laboratory of Image and Signal Processing (KULIS) at Kocaeli University. Her research interests focus on image processing, data fusion on remotely sensed data and hyperspectral imaging.

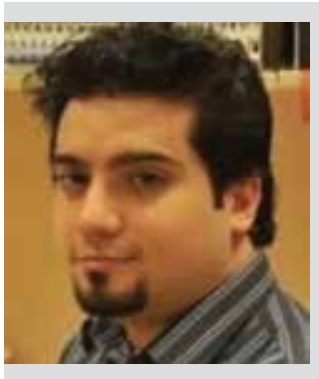

Alp Ertürk received his B.Sc. and M.Sc. degrees in Electrical and Electronics Engineering from Middle East Technical University, Ankara, Turkey, in 2007 and 2010, respectively. He received his Ph.D. degree in Electronics and Telecom. Engineering from Kocaeli University, Kocaeli, Turkey, in 2013. He was a postdoctoral researcher with the Hyperspectral Computing Laboratory (HyperComp) in University of Extremadura, Cáceres, Spain. He is currently an Assistant Professor with the Electronics \& Telecom. Eng. Department, Kocaeli University, Turkey. His research interests are in the areas of digital image processing, remote sensing and hyperspectral imaging. $\mathrm{He}$ is a reviewer for several journals including IEEE Geoscience and Remote Sensing (GRSL), IEEE Journal of Selected Topics in Applied Earth Observations and Remote Sensing (JSTARS) and IEEE Transactions of Geoscience and Renote Sensing (TGRS). He is also currently serving as the Secretary and Treasurer of the IEEE GRSS Turkey Chapter. 